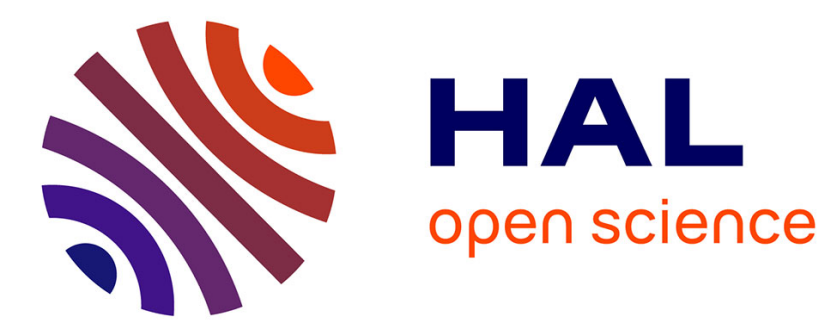

\title{
Directed diffusion-controlled aggregation versus directed animals
}

J. Nadal, Bernard Derrida, J. Vannimenus

\section{To cite this version:}

J. Nadal, Bernard Derrida, J. Vannimenus. Directed diffusion-controlled aggregation versus directed animals. Physical Review B, 1984, 30 (1), pp.376-383. 10.1103/PhysRevB.30.376 . hal-03285924

\section{HAL Id: hal-03285924 \\ https://hal.science/hal-03285924}

Submitted on 19 Jul 2021

HAL is a multi-disciplinary open access archive for the deposit and dissemination of scientific research documents, whether they are published or not. The documents may come from teaching and research institutions in France or abroad, or from public or private research centers.
L'archive ouverte pluridisciplinaire HAL, est destinée au dépôt et à la diffusion de documents scientifiques de niveau recherche, publiés ou non, émanant des établissements d'enseignement et de recherche français ou étrangers, des laboratoires publics ou privés. 


\title{
Directed diffusion-controlled aggregation versus directed animals
}

\author{
J. P. Nadal \\ Groupe de Physique des Solides de l'Ecole Normale Supérieure, 24 rue Lhomond, 75231 Paris Cedex 05, France \\ B. Derrida \\ Service de Physique Théorique, Centre d'Etudes Nucléaires-Saclay, 91191 Gif-sur-Yvette, France \\ J. Vannimenus \\ Groupe de Physique des Solides de l'Ecole Normale Supérieure, 24 rue Lhomond, 75231 Paris Cedex 05, France
}

(Received 28 November 1983)

\begin{abstract}
Monte Carlo calculations are performed for two directed systems: the problem of directed animals and the problem of directed aggregation. Taking advantage of recent exact results obtained for the directed animal model, we present a Monte Carlo method which produces directed animals with unbiased statistics, in contrast with previous methods for polymer problems. Large typical clusters of each type are displayed and estimations of the exponents governing the mean length and width of the clusters are obtained, showing that the two models are in different universality classes.
\end{abstract}

\section{INTRODUCTION}

Recently there has been much interest in the study of kinetic growth of clusters by statistical physics methods. The processes involved are related to the growth of crytals, dendrites, ${ }^{1}$ tumors, ${ }^{2}$ and to the coagulation of small particles as smoke, dust, and aggregation of colloids. ${ }^{3-9}$ In these latter cases, the cluster grows through the aggregation of a particle coming from far away by a diffusion process. The aggregate is characterized in particular by its Hausdorff dimension $D$, giving the mean radius $R$ of a cluster of mass $s$ (number of sites in the cluster):

$$
R \sim s^{v}, \quad v=\frac{1}{D} .
$$

$D$ is found to be about 1.7 in two dimensions (2D's), ${ }^{3,4} 2.4$ in 3D's and 3.3 in 4D's (Ref. 4). These results and others $^{5-9}$ indicate that these models are not in the same universality class as other geometrical models, as percolation, ${ }^{10}$ linear polymers, ${ }^{11}$ or branched polymers (animals). ${ }^{10}$

On the other hand, recently much work has also been done on directed random cluster systems, as directed percolation $^{12}$ and directed animals. ${ }^{13-18}$ These models take into account the effect of a strong external field, such as a gravitational or electric field: for example, directed percolation has been proposed as a model for the hopping conductivity in a strong electric field ${ }^{19}$ and directed animals for the shape of a river network. ${ }^{16}$ As in the nondirected case, it is interesting to study what happens when the cluster is grown by a kinetic process. The processes involved may be a reaction of polymerization inside a flow of solution containing the monomers, the capture of small aerosol particles on fibers ${ }^{20}$ or surfaces ${ }^{21}$ in the presence of a strong electric field, or the pattern formed by electric breakdown in an insulator. ${ }^{22}$

In this paper we want to compare two two-dimensional (2D) lattice-directed systems, described hereafter, one of directed kinetic growth and one of directed animals. The two models are chosen such that the allowed clusters are exactly the same (but the statistics, i.e., the weights of the clusters, are different in the two models; see Fig. 1). We study here systems of site clusters by means of Monte Carlo methods on a square lattice and on strips of finite width.

In Sec. II we present the two models and give for both of them some pictures of typical clusters. In Sec. III, the Monte Carlo methods used for each model are explained. Our results are described and discussed in Sec. IV.

\section{MODELS}

We first recall that a directed cluster is defined by the following property: ${ }^{12}$ there exists a "root" or a "seed"that is, a given set of sites of the cluster-such that any point of the cluster can be reached from the seed via a

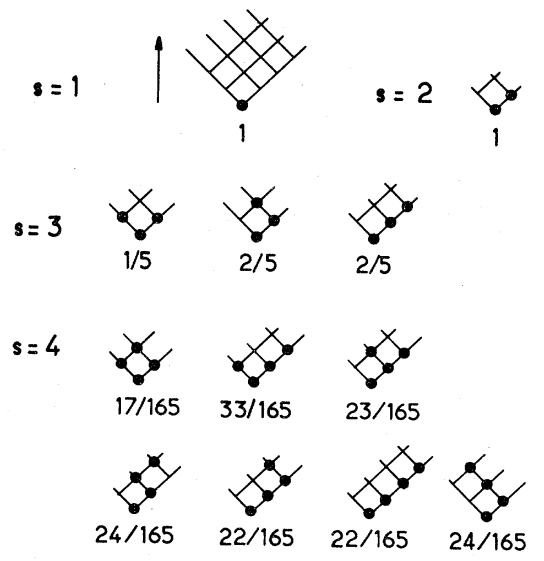

FIG. 1. Directed clusters with $s$ sites, for $s \leq 4$. The preferred direction is shown by the arrow. The seed is the lowest site of the cluster. The weights of the clusters for the aggregation model are shown under each cluster. For $s \leq 3$, they are the same as for the animal model, but for $s \geq 4$ the weights are different. For $s=4$, there are 13 clusters, such that each directed animal has the same weight $\frac{1}{13}$. We have identified symmetric clusters; so, for example, for $s=4$, in the directed-animal model, the first cluster has a weight $\frac{1}{13}$, and each of the others has a weight $\frac{2}{13}$. 
path which never goes opposite to a given preferred direction. The shape of such a cluster with a large size $s$ can be described by two different exponents $v_{\|}$and $v_{\perp}$ (instead of one $v=1 / D$ ), giving the behavior of the mean length $L$ and width $W$ :

$$
s \rightarrow \infty, L \sim s^{v_{\|}}, W \sim s^{v_{\perp}} .
$$

\section{A. Directed animals}

In the problem of directed animals one gives the same weight to each allowed cluster. Here we consider the model of directed-site animals on the 2D square lattice, with the preferred direction lying along a diagonal (model $A$ of Ref. 17). For this model some exact results have been obtained, ${ }^{13,17,18}$ giving in particular ${ }^{18}$ the exact number $\Omega_{s}(\Gamma)$ of directed animals of $s$ sites with a root $\Gamma, \Gamma$ being a given set of occupied sites on a row orthogonal to the preferred direction, and the exact value $v_{\perp}=\frac{1}{2}$. However, the exact value of $v_{\|}$is not known, and the following values have been obtained up to now: $v_{\|}=0.800 \pm 0.001$ (by direct enumeration ${ }^{14}$ ), $v_{\|}=0.8$ (field theory and Pade approximants, ${ }^{15} v_{\|}=0.818 \pm 0.001$ phenomenological renormalization ${ }^{17}$ ). Figure 2 shows some typical directed animals obtained by a Monte Carlo method explained in Sec. III. These animals are of sizes 500, 1000, and 2000.

\section{B. Directed diffustion-controlled aggregation}

The model is a simple generalization of the model first proposed by Witten and Sander ${ }^{3}$ shortly described in the Introduction: given a seed, a particle diffuses and stops if it reaches a site adjacent to tive seed; then a new particle is launched, and so on. Here we introduce a preferred direction in the diffusion process: the particle "falls" from far above the cluster and is halted when adjacent to one site of the cluster. As for the directed-animal model, the preferred direction lies along the diagonal of the square lattice and a seed is set at the origin. Figure 3 shows a typical aggregate at three stages of its growth, that is with 1000,5000 , and 10000 particles.

\section{MONTE CARLO METHODS}

\section{A. Directed animals}

It is usually a difficult problem to study linear or branched polymers by Monte Carlo methods. Indeed, there is no efficient Monte Carlo method which produces long polymers with the correct probability. Known methods introduce a systematic bias and many algorithms have been proposed to make this bias as small as possible. $^{23}$

For a model of directed animals, because we know the exact expression for the number $\Omega_{S}(\Gamma)$ of animals of any given number of sites $S$ and any given root $\Gamma,{ }^{18}$ we can use a Monte Carlo method that does not suffer from any bias. The idea is to construct row by row a typical directed animal of $S$ sites. Let us suppose we have already constructed the cluster up to $N$ sites and to a distance $L$ from
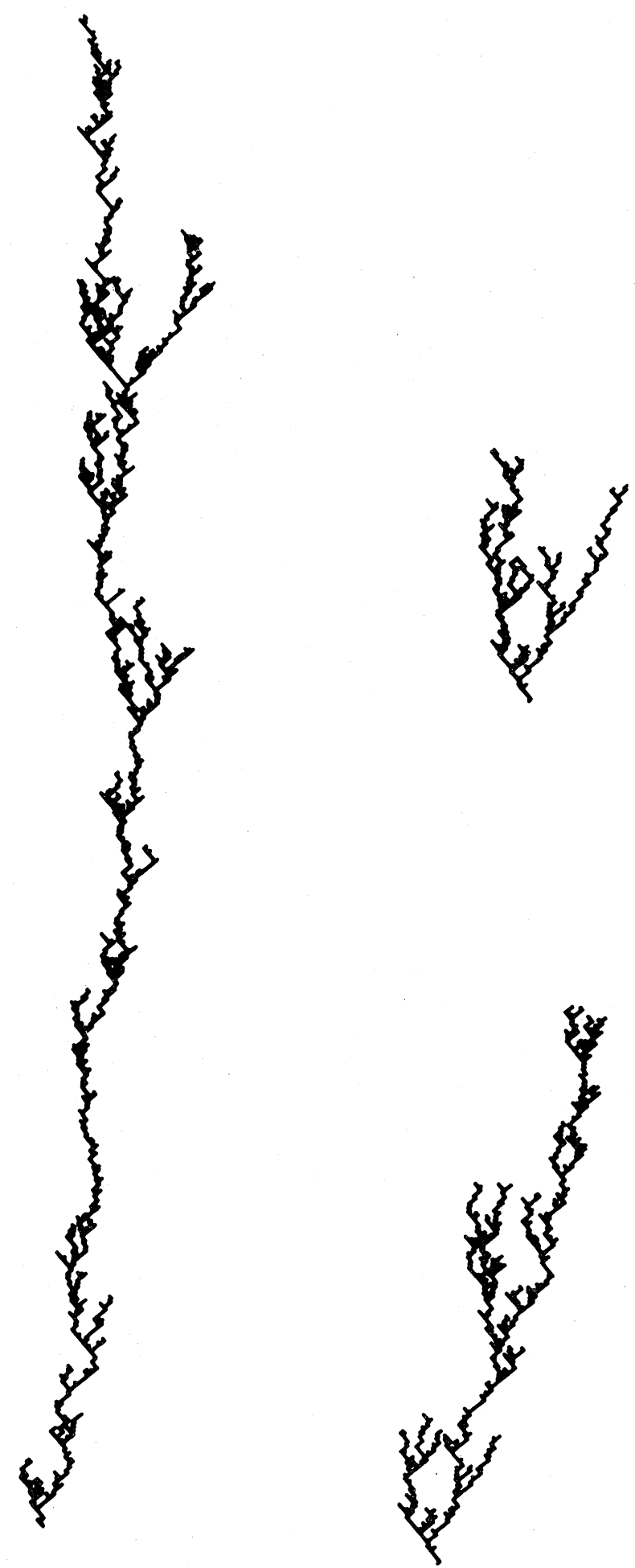

FIG. 2. Typical directed animals obtained by a Monte Carlo method. These animals are of sizes 500, 1000, and 2000.

the root. We want to add a new row. Let $\Gamma$ be the configuration of occupied sites on the $L$ th row. On the $(L+1)$ th row one can construct any configuration $\Gamma^{\prime \prime}$ of occupied sites such that any site of $\Gamma^{\prime \prime}$ is connected to a least one occupied site of $\Gamma$. Among all these possible configurations $\Gamma^{\prime \prime}$, we must pick one of them, e.g., $\Gamma^{\prime}$, 

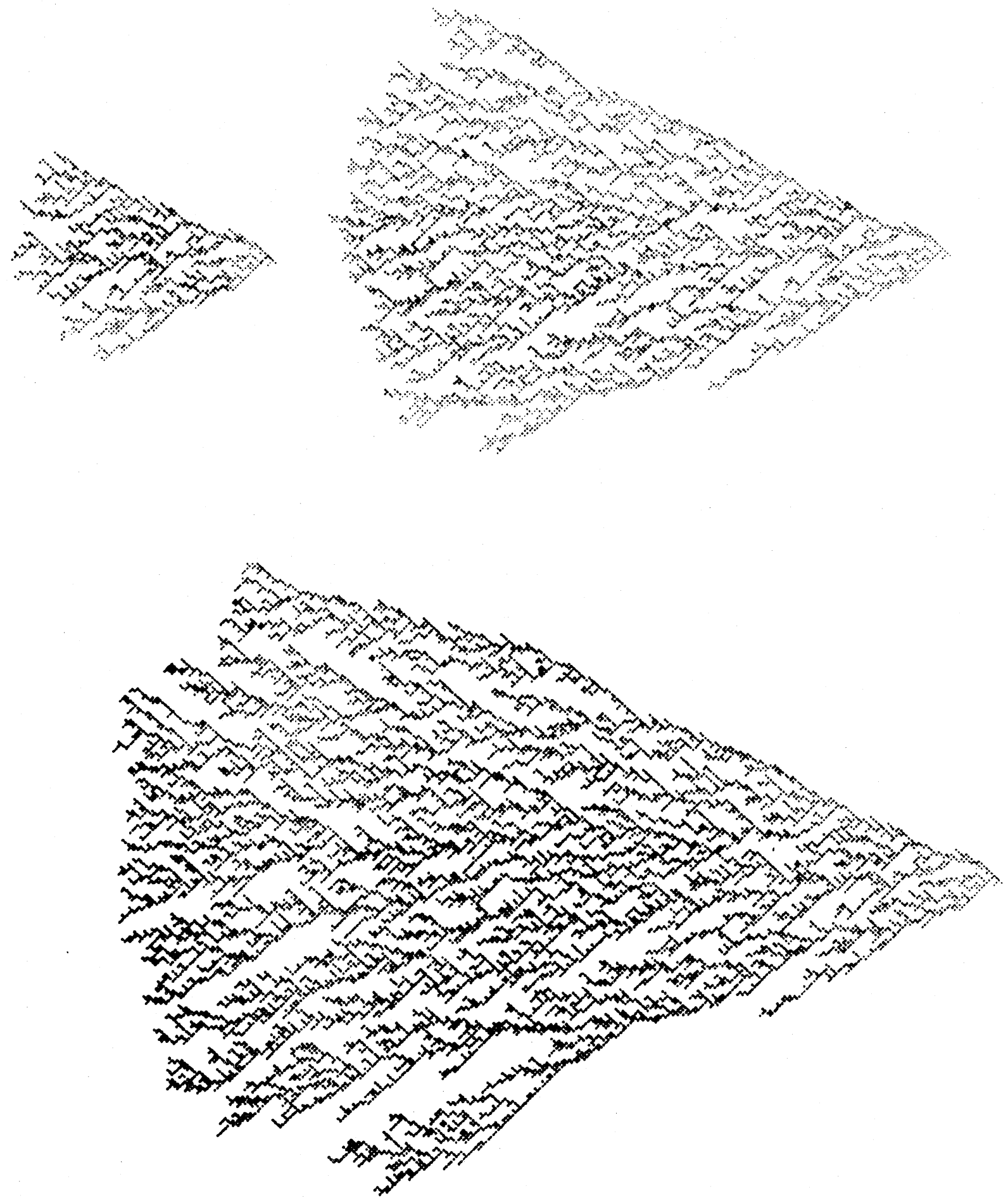

FIG. 3. Typical directed aggregate at three stages of its growth: 1000, 5000, and 10000 particles.

with a probability $P\left(\Gamma^{\prime}\right)$. This probability $P\left(\Gamma^{\prime}\right)$ must be chosen to give the same weight to all configurations of $S$ sites which coincide with the part of the cluster already constructed (columns $1-L$ ), that is

$$
P\left(\Gamma^{\prime}\right)=\frac{\Omega_{S-N}\left(\Gamma^{\prime}\right)}{\sum_{\Gamma^{\prime \prime}}^{\prime} \Omega_{S-N}\left(\Gamma^{\prime \prime}\right)}=\frac{\Omega_{S-N}\left(\Gamma^{\prime}\right)}{\Omega_{S-N+m}(\Gamma)},
$$

where $\Sigma^{\prime}$ denotes the sum over all the configurations $\Gamma^{\prime \prime}$ which can follow $\Gamma$, and $m=m(\Gamma)$ is the number of occupied sites at column $L$.

The expression for all $\Omega_{s}(\Gamma)$ is known ${ }^{18(a)}$ for animals on strips of finite width $n$, then one can easily obtain the expression for the 2D lattice [the notations are those of Refs. 17 and 18(a)]: 


$$
\begin{aligned}
\Omega_{s}(\Gamma)=\int_{0}^{2 \pi} & \frac{d k}{\pi} \cos \frac{k}{2} \cos k\left(r-\frac{1}{2}\right)(1+2 \cos k)^{s-1} \\
& \times \prod_{j>0}\left[\frac{\sin \left(j+\frac{1}{2}\right) k}{\sin (k / 2)}\right]^{N_{j}}
\end{aligned}
$$

( $r$ is the width of the root $\Gamma ; \Gamma$ is denoted $\mathscr{C}$ in Ref. 17). However, from a practical point of view, one can note that for $n>S$ the values on the strip and on the 2D lattice are the same and $\Omega_{s}$ can be expressed as a sum rather than an integral.

Even though the exact expression of all $\Omega_{S-N}(\Gamma)$ is known, the calculation of the probabilities $P\left(\Gamma^{\prime}\right)$ is a long task since one must evaluate these probabilities for all the possible $\Gamma^{\prime}$. To avoid this long calculation at each step of the construction of the cluster we have used the method of importance sampling: ${ }^{24}$ one chooses $\Gamma^{\prime}$ with some given probability $Q\left(\Gamma^{\prime}\right)$ which is simple to evaluate; then this choice is accepted with a probability $r\left(\Gamma^{\prime}\right)$, so the probability to effectively obtain $\Gamma^{\prime}$ is

$$
\begin{aligned}
\hat{P}\left(\Gamma^{\prime}\right)= & Q\left(\Gamma^{\prime}\right) r\left(\Gamma^{\prime}\right)\left[1+\left[\sum_{\Gamma^{\prime \prime}}^{\prime} Q\left(\Gamma^{\prime \prime}\right)\left[1-r\left(\Gamma^{\prime \prime}\right)\right]\right]\right. \\
& \left.+[]^{2}+\cdots\right] \\
= & \frac{Q\left(\Gamma^{\prime}\right) r\left(\Gamma^{\prime}\right)}{\sum_{\Gamma^{\prime \prime}}^{\prime} Q\left(\Gamma^{\prime \prime}\right) r\left(\Gamma^{\prime \prime}\right)}
\end{aligned}
$$

We want $\hat{P}\left(\Gamma^{\prime}\right)$ to be equal to $P\left(\Gamma^{\prime}\right)$. Therefore $r\left(\Gamma^{\prime}\right)$ must be given by

$$
r\left(\Gamma^{\prime}\right)=A \frac{P\left(\Gamma^{\prime}\right)}{Q\left(\Gamma^{\prime}\right)} .
$$

$A$ is an arbitrary constant to be taken such that, for any $\Gamma, r(\Gamma)$ is less than 1 but as large as possible. The main advantage of this importance sampling method is that we must calculate $P\left(\Gamma^{\prime}\right)$ only for one configuration $\Gamma^{\prime}$ instead of needing to calculate it for all configurations $\Gamma^{\prime \prime}$ as in formula (2).

We use here a simple choice for $Q\left(\Gamma^{\prime}\right)$ : For each site on the $(L+1)$ th row which is allowed to be occupied, we choose it occupied with a probability $p$ and empty with a probability $(1-p)$. Then

$$
Q\left(\Gamma^{\prime}\right)=p^{m\left(\Gamma^{\prime}\right)}(1-p)^{n\left(\Gamma^{\prime}\right)-m\left(\Gamma^{\prime}\right)},
$$

where $n\left(\Gamma^{\prime}\right)$ is the number of sites which can be occupied on a row following $\Gamma$ and $m\left(\Gamma^{\prime}\right)$ is the number of occupied sites in $\Gamma^{\prime}$. The final results do not depend on the particular values of $A$ and $p$ used, but good choices increase the efficiency of the method-note that the mean number of trials before accepting a new configuration is $1 / A$.

\section{B. Directed aggregation}

It is easy in this case to perform a Monte Carlo study. If the seed is a single site, the cluster will be confined in a cone starting from the seed, of half angle $\pi / 4$, and with the preferred direction as the axis. If $L$ is the height of the cluster measured along this axis, one launches a particle at height $L+1$ from a point chosen at random with equal probability for every point at this height in the cone.
If the particle gets out of the cone, it is lost, and another one is launched. At each diffusion step the particle can choose between two sites under it; if at least one of them is occupied, the particle is halted.

\section{RESULTS}

\section{A. Monte Carlo on strips}

The methods described above can be performed on systems confined on strips of finite width $n$ with periodic boundary condition, and of infinite length parallel to the preferred direction. On such a strip one can calculate the average length $\langle L\rangle$ of a cluster of $s$ sites, which for large $s$ is expected to behave in the following way:

$$
\langle L\rangle \sim n^{\left(v_{\|}-1\right) / v_{\perp}} s .
$$

An argument for this relation, which generalizes the one originally used for polymers, ${ }^{25}$ is given in Ref. 17 .

In Table $\mathrm{I}(\mathrm{a})$ the values of $\langle L\rangle / s$ for the aggregation model are given, and Fig. 4 shows $\ln \langle L\rangle / s$ vs $\ln n$. For directed aggregation we obtain the value

TABLE I. Values of $\langle L / s\rangle$ for strips of different width $n$. (a): Directed aggregation. The value at $n=2$ is an exact numerical value. We have obtained this value by a method which is rather complicated and which we shall not describe here since we could not generalize it to $n \geq 3$. (b): Directed animals. The values for $n \leq 13$ are the exact values obtained in Ref. 17 .

\begin{tabular}{rl}
\hline$n$ & \multicolumn{1}{c}{$\langle L / s\rangle$} \\
2 & $($ a) \\
20 & 0.72576 \\
30 & $0.1280 \pm 0.0001$ \\
40 & $0.0651 \pm 0.0001$ \\
50 & $0.0522 \pm 0.0001$ \\
60 & $0.0438 \pm 0.0001$ \\
70 & $0.0378 \pm 0.0001$ \\
100 & $0.0263 \pm 0.0001$ \\
200 & $0.0135 \pm 0.0002$ \\
& \\
& \\
2 & 1.17157 \\
3 & 1.34405 \\
4 & 1.48783 \\
5 & 1.61139 \\
6 & 1.72052 \\
7 & 1.81883 \\
8 & 1.90871 \\
9 & 1.99179 \\
10 & 2.06925 \\
11 & 2.14199 \\
12 & 2.21067 \\
13 & 2.27584 \\
15 & $2.40 \pm 0.02$ \\
20 & $2.66 \pm 0.02$ \\
30 & $3.13 \pm 0.04$ \\
\hline \hline
\end{tabular}



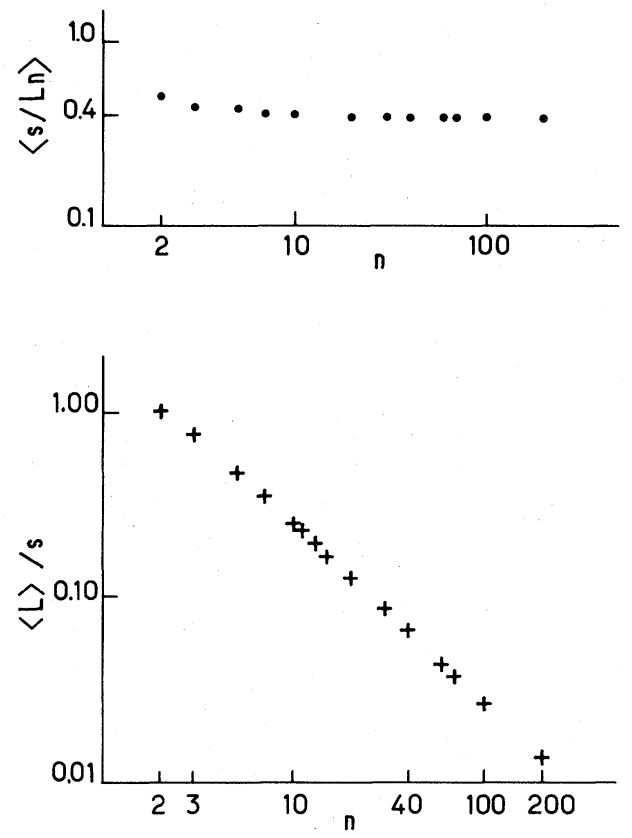

FIG. 4. Monte Carlo on strips for directed aggregation. On top, density $\rho(n)=\langle s / L n\rangle$ for large $s$; at the bottom $\langle L / s\rangle$ vs $n$ in a log-log plot.

$$
\frac{1-v_{\|}}{v_{\perp}}=0.99 \pm 0.01
$$

In any case we expect $v_{\|}+v_{\perp} \geq 1$, since there is at most one particle per lattice site, and the equality would mean that the cluster has a finite density $\rho$ for large $s$. Indeed, the drawings in Fig. 3 are in agreement with a value $v_{\|}+v_{1}$ close to 1 -otherwise one would expect to see holes of any sizes in the cluster. The values $\rho_{n}=\langle s / L n\rangle$ are also shown in Fig. 4. If we assume that $v_{\|}+v_{\perp}=1$, for the density we obtain

$$
\begin{aligned}
& \rho=\lim _{\substack{s \rightarrow \infty \\
n \rightarrow \infty}}\langle s / \operatorname{Ln}\rangle, \\
& \rho=0.384 \pm 0.005 .
\end{aligned}
$$

The compactness of the clusters is in agreement with similar calculations made independently by Jullien et al. ${ }^{26}$ and by Meakin. ${ }^{27}$ Jullien et al. analyzed the behavior of $\langle L\rangle$ using only one exponent, $\langle L\rangle \sim n^{1-D} s$, from which they obtained $D \sim 2$.

For directed animals we already have from Ref. 17 the (exact) values of $b_{n}=\langle L\rangle / s$ for $n \leq 13$. We made Monte Carlo calculations for larger strips $(n=15,20$, and 30) [Table $\mathbf{I}(\mathbf{b})$ ]. We tried an analysis assuming a form $\langle L / s\rangle_{(n)}=A n^{a}+B n^{a-b}$ where $a$ is interpreted as $a=\left(1-v_{\|}\right) / v_{\perp}$. Taking into account only the values for $n \leq 13$, one can extrapolate the possible values for $n=15$ and 20 for different values of $b$. If $b$ is not too small $(b>1.0)$ then these values are not very sensitive to $b$ (for $n=20$, the possible values of $\langle L / s\rangle$ are between 2.657 and 2.667), and within the error bars of the Monte Carlo calculations. As a result, $v_{\|}$takes a value between 0.816 and 0.819 [with $n \leq 13$, the value 0.818 (Ref. 17) is ob- tained for $b=2]$. To obtain a value of $v_{\|}$of order 0.800 (as proposed in Ref. 14), one must choose a very low value for the correction exponent $b$, and to accept the highest values of $\langle L / s\rangle$ compatible with the error bars $(\langle L / s\rangle>2.68$ for $n=20)$.

\section{B. Results for $2 \mathrm{D}$ systems}

For clusters generated on the 2D square lattice we calculate different moments of the distribution of lengths and widths of the clusters.

(i) The mean length $\langle L\rangle$ and width $\langle w\rangle$, and expect for large $s$

$$
\langle L\rangle \sim s^{v_{\|}},\langle w\rangle \sim s^{v_{\perp}} .
$$

(ii) Let $X_{i}, Y_{i}$ denote the coordinates of the $i$ th particle in the cluster $(i=1, s)$; the seed $(i=1)$ is set at the origin $\left(X_{1}=Y_{1}=0\right)$ and $X_{i}, Y_{i}$ are, respectively, measured orthogonally to the preferred direction and along it. We define, for $Z$ being $X$ or $Y$,

$$
\mu_{p}(Z)=\left(\frac{1}{s} \sum_{i=1}^{S} Z_{i}^{p}\right)^{1 / p}, m_{2}(Z)=\left(\mu_{2}^{2}-\mu_{1}^{2}\right)^{1 / 2},
$$

and one expects for large $s$

$$
\begin{aligned}
& \left\langle\mu_{1}(Y)\right\rangle \sim\left\langle\mu_{2}(Y)\right\rangle \sim\left\langle m_{2}(Y)\right\rangle \sim s^{v_{\|}}, \\
& \left\langle\mu_{2}(X)\right\rangle \sim\left\langle m_{2}(X)\right\rangle \sim s^{v_{\perp}}, \\
& \left\langle\mu_{1}(X)\right\rangle=0 .
\end{aligned}
$$

\section{Directed aggregation}

For this model, these quantities are shown in Fig. 5, for $1000 \leq s \leq 18000$, the average being taken over 7 samples. The slopes compatible with these data lead to the following estimates:

$$
\begin{aligned}
& v_{||}=0.52 \pm 0.02, \\
& v_{\perp}=0.49 \pm 0.02 .
\end{aligned}
$$

This is in reasonable agreement with the strip calculations-if one takes $v_{\|}=0.52, v_{\perp}=0.49$, then $\left(1-v_{\|}\right) / v_{\perp} \simeq 0.98$-we note that there is the possibility that $v_{\|}=v_{\perp}=\frac{1}{2}$, or the weaker relation $v_{\|}+v_{\perp}=1$, which can be justified by the following heuristic argument.

It is found in the simulations that the maximum width of the aggregate is reached near its top, so that it has roughly the shape of a triangle of height $L$ and width $W$. The particles start with uniform probability from any of the $2 L$ points lying in the allowed cone, at a height $L+1$ above the root: those particles starting just above the triangle are very likely to reach the cluster, and there is in addition a region of width $L^{1 / 2}$ where particles have a finite probability to be captured during their diffusion. If we assume that $W \gg L^{1 / 2}$, as observed, the probability that the cluster mass increases by one unit is

$$
P(\Delta N=1) \sim W / L .
$$

Now the probability that the height increases by one 

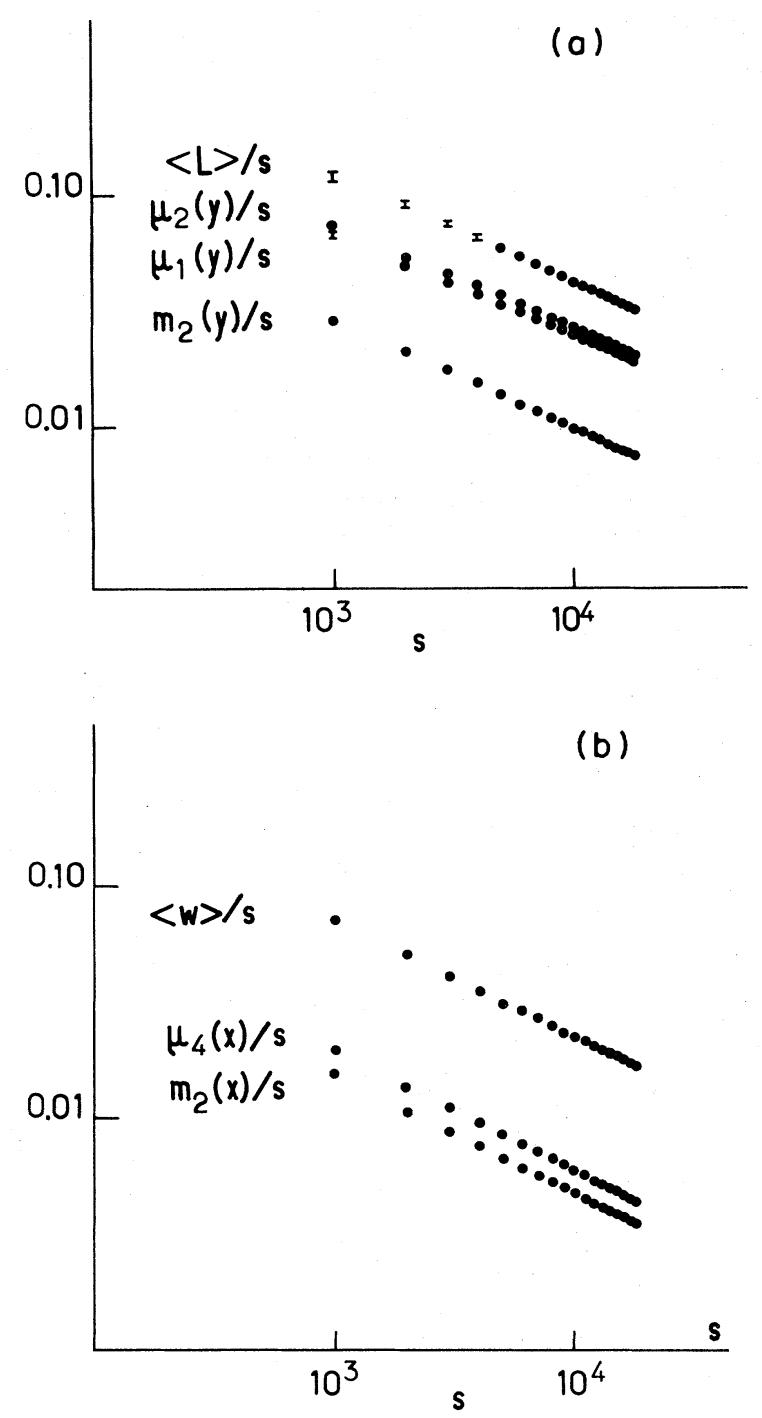

FIG. 5. (a) Results for 2D directed aggegrates: The various curves are expected to have slope $\left(v_{\|}-1\right)$ for large $s$ $(1000 \leq s \leq 18000)$. (b) Results for $2 \mathrm{D}$ directed aggregates: The slopes are expected to be equal to $v_{1}-1$ for large $s$.

unit is related to the average number of topmost cluster sites. If we assume that this number does not increase with $N$, there comes

$$
P(\Delta L=1) \sim L^{-1},
$$

and therefore

$$
\frac{\langle\Delta L\rangle}{\langle\Delta N\rangle} \sim W^{-1}
$$

which gives

$$
\begin{aligned}
& N^{v_{\|}-1} \sim N^{-v_{\perp}}, \\
& \text { i.e., } \\
& v_{\|}+v_{\perp}=1 .
\end{aligned}
$$

If the further assumption is made that the number of sites at the maximum distance from the axis remains fin- ite when $N$ increases, one obtains along similar lines

$$
\frac{\langle\Delta W}{\langle\Delta N\rangle} \sim W^{-1},
$$

which gives $v_{\perp}=\frac{1}{2}$.

\section{Directed animals}

We realized a detailed statistical study for animals of sizes smaller than 150. The results are displayed in Fig. 6. For $v_{\perp}$, our data lead to the following estimate:

$$
v_{\perp}=0.49 \pm 0.03
$$

which is in good agreement with the exact value $v_{\perp}=\frac{1}{2}$. A direct measure of the slopes in Fig. 6 leads to an estimate of $v_{\|}=0.85 \pm 0.01$ for the mean length $\langle L\rangle$. However, if we assume a behavior of the type $\langle L\rangle \sim S^{v_{\|}}\left(A+B s^{-\omega}\right)$ which takes into account corrections to scaling, we find

$$
v_{\|}=0.824 \pm 0.015 \text {. }
$$

For $\mu_{2}(Y)$ an analysis of the same type gives
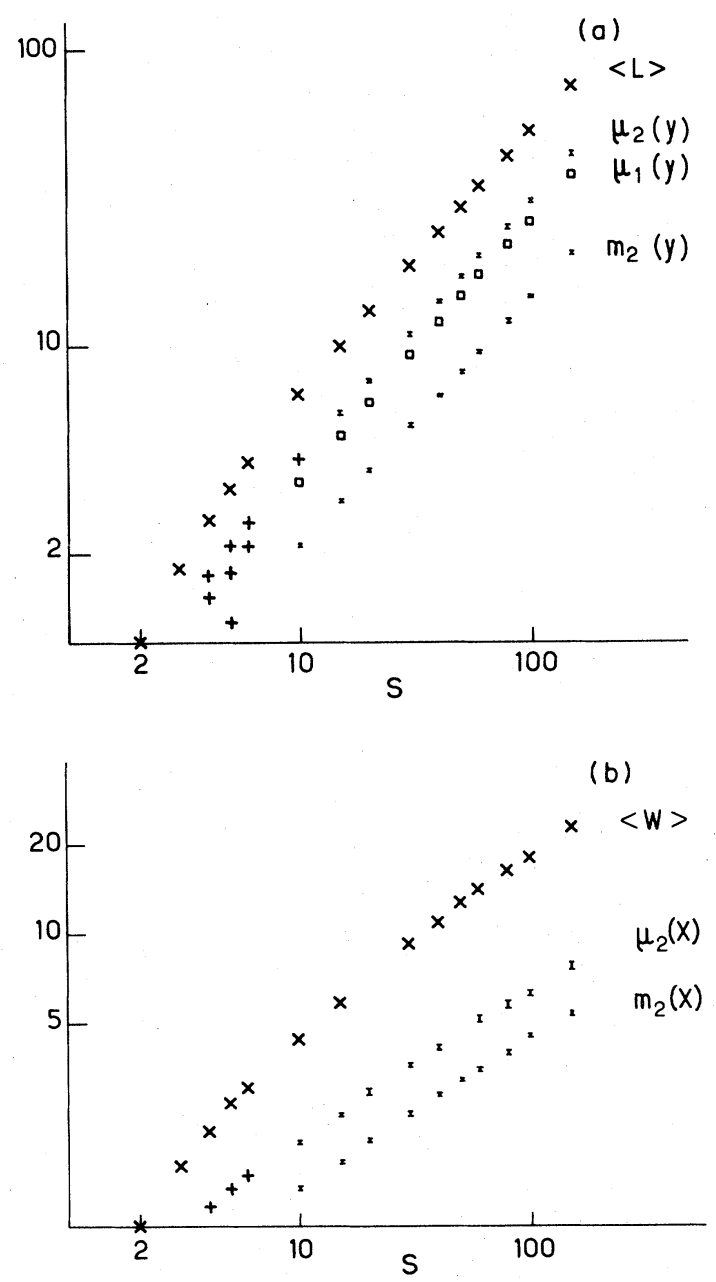

FIG. 6. (a) Results for 2D directed animals. The slopes are expected to be equal to $v_{\|}$. (b) Results for 2D directed animals. The slopes are expected to be equal to $v_{\perp}$. 
$v_{\|}=0.83 \pm 0.02$. These estimations of $v_{\|}$by the Monte Carlo method favors the conjectured value $v_{\|}=\frac{9}{11}$ rather than 0.800 .

\section{Fractal dimension}

Let us now discuss the question of the fractal dimension $D$ of directed clusters. Suppose that we have constructed a large directed cluster of $s$ sites and that we want to measure its fractal dimension $D$. The canonical procedure is to use squares of linear size $\epsilon$ and to calculate the minimum number $\mathscr{N}(\epsilon)$ of squares which are necessary to cover the cluster. In the regime $\epsilon \gg 1$ and $\epsilon$ much smaller than $s^{v_{\|}}$and $s^{v_{\perp}}$, one expects $\mathscr{N}(\epsilon)$ to behave similar to

$$
\mathscr{N}(\epsilon) \sim \epsilon^{-D} .
$$

In practice it is difficult to produce a sufficiently large cluster to satisfy the condition $1<<\epsilon<s^{v_{\perp}}$. Therefore, we have used several approximate definitions of this fractal dimension $D$. The first one consists in choosing $\epsilon$ of the order of the smallest of $s^{v_{\|}}$and $s^{\nu_{\perp}}$, which leads to

$$
D^{\prime}=1+\frac{1-v_{\|}}{v_{\perp}}
$$

for $v_{\perp}<v_{\|}$. This definition is reasonable since it gives $D=1$ for directed self-avoiding walks. Moreover, if we compare with Eq. (7), it gives that for a strip of width $n$

$$
\langle L / s\rangle \sim n^{1-D},
$$

a formula which is valid for nondirected clusters. ${ }^{25}$ For directed animals in $2 \mathrm{D}$ with $\nu_{1}=\frac{1}{2}$ and $v_{\|} \simeq \frac{9}{11}$ one obtains

$$
D^{\prime} \sim 1.38,
$$

and for directed aggregation

$$
D^{\prime} \sim 1.99 \pm 0.01 \text {. }
$$

Let us note that if $v_{\perp}>v_{\|}$, one should replace formula (19) by

$$
D^{\prime}=\frac{1}{v_{\|}}+(d-1)\left(1-\frac{v_{\perp}}{v_{\|}}\right) .
$$

Another approximate definition of $D$ consists in writing that the total mass $s$, as a function of the macroscopic volume of the cluster, behaves similar to

$$
s \sim\left(L W^{d-1}\right)^{D^{\prime \prime} / d}
$$

with

$$
D^{\prime \prime}=\frac{d}{(d-1) v_{\perp}+v_{\|}} .
$$

All these quantities, $D, D^{\prime}$, and $D^{\prime \prime}$, give, for $v_{\|}=v_{\perp}=v$, the proper fractal dimension of a nondirected system, $D=1 / v$. However, the fractal dimension $D$ gives a rough characterization of directed clusters, since one knows that they are characterized by two critical exponents, $v_{\|}$and $v_{1}$.

\section{CONCLUSION}

In this paper we performed Monte Carlo calculations for two directed systems, one of directed animals and one of directed aggregation. Whereas in the two models the possible clusters are the same, the typical clusters here obtained show the difference between the two statistics. This difference is illustrated by the calculation, on strips of finite width and on 2D systems, of the exponents which govern the length and the width of the clusters with a large number of particles. For the directed-animal model, the results are in good agreement with our previous work, giving a value for $v_{\|}$of about 0.82 . For directed aggregation, $v_{\|}$and $v_{\perp}$ are both close to $\frac{1}{2} \quad\left(v_{\|}=0.52 \pm 0.02\right.$, $v_{\perp}=0.49 \pm 0.02$ ), which indicates that the model is also in a different universality class than the (nondirected) aggregation models recently studied by various authors.

\section{ACKNOWLEDGMENTS}

We thank P. Y. Le Daëron and L. De Seze for their help in realizing the figures of the clusters, and $H$. J. Herrmann and M. H. Kalos for interesting discussions. We are also indebted to R. Jullien, R. Botet, and M. Kolb for communicating their own work prior to publication.
1P. A. Rikvold, Phys. Rev. A 26, 647 (1982).

${ }^{2}$ M. Eden, in Proceedings of the Fourth Berkeley Symposium on Mathematical Statistics and Probability, edited by J. Neyman (University of California Press, Berkeley, 1961), Vol. IV, p. 223; H. P. Peters, D. Stauffer, H. P. Hölters, and K. Loewenich, Z. Phys. B $\underline{34}, 399$ (1979).

${ }^{3}$ T. A. Witten and L. M. Sander, Phys. Rev. Lett. 47, 1400 (1981); Phys. Rev. B 27, 5686 (1983).

${ }^{4}$ P. Meakin, Phys. Rev. A 27, 1495 (1983); 27, 604 (1983); J. M. Deutch and P. Meakin, J. Chem. Phys. $\underline{78}, 2093$ (1983).

${ }^{5}$ M. Muthukumar, Phys. Rev. Lett. 므, 839 (1983).

${ }^{6}$ H. Gould, F. Family, and H. E. Stanley, Phys. Rev. Lett. $\underline{50}$, 686 (1983); M. Sahimi and G. R. Jerauld, J. Phys. A 16, L419 (1983).
${ }^{7}$ T. A. Witten (unpublished).

${ }^{8}$ M. Nauenberg, R. Richter, and L. M. Sander, Phys. Rev. B 28 , 1649 (1983); M. Nauenberg and L. M. Sander, Physica 123A, 360 (1984); M. Nauenberg, Phys. Rev. B 28, 449 (1983); L. M. Sander, Z. M. Cheng, and R. Richter ibid. 28, 6394 (1983).

${ }^{9}$ H. E. Stanley, F. Family, and H. Gould (unpublished)

${ }^{10}$ D. Stauffer, in Disordered Systems and Localization, Vol. XX of Lecture Notes in Physics, edited by C. Castellani, C. Di Castro, and L. Peliti (Springer, Berlin, 1981), p. 9.

${ }^{11}$ D. S. McKenzie, Phys. Rep. C $\underline{27}, 35$ (1972).

${ }^{12}$ W. Kinzel, in Percolation Structures and Processes, edited by G. Deutscher, R. Zallen, and J. Adler (Hilger, Bristol, 1982).

${ }^{13}$ D. Dhar, M. K. Phani, and M. Barma, J. Phys. A 15, L279 (1982); D. Dhar, Phys. Rev. Lett. 49, 959 (1982). 
${ }^{14}$ S. Redner and Z.-R. Yang, J. Phys. A 15, L177 (1982); H. E. Stanley, S. Redner, and Z.-R. Yang, ibid. 15, L569 (1982).

${ }^{15}$ N. Breuer and H. K. Janssen, Z. Phys. B $\underline{48}, 347$ (1982); J. L. Cardy, J. Phys. A 15, L593 (1982).

16J. E. Green and M. A. Moore, J. Phys. A 15, L597 (1982).

${ }^{17}$ J. P. Nadal, B. Derrida, and J. Vannimenus, J. Phys. (Paris) $\underline{43}, 1561$ (1982).

18(a) V. Hakim and J. P. Nadal, J. Phys. A 16, L213 (1983); (b) D. Gouyou-Beauchamp and G. Viennot (unpublished); D. Dhar, Phys. Rev. Lett. 51, 853 (1983).

${ }^{19}$ M. Van der Meer, R. Keiper, and R. Schuchardt, J. Phys. (Paris) Colloq. C 4, Suppl. 10, 42, 175 (1981); N. Van Lien and B. I. Shklovskii, Solid State Commun. 38, 99 (1981).

${ }^{20}$ M. J. Oak and D. A. Saville, J. Colloid Interface Sci. 6ㅡ, 259 (1980).

${ }^{21}$ P. Meakin, Phys. Rev. A $\underline{27}, 2616$ (1983).

${ }^{22}$ Y. Sawada, S. Ohta, M. Yamazaki, and H. Honjo, Phys. Rev. A $\underline{26}, 3557$ (1982).
${ }^{23}$ I. Webman, J. L. Lebowitz, and M. H. Kalos, Macromolecules 14, 1495 (1981); J. Tobochnik, I. Webman, J. L. Lebowitz, and M. H. Kalos, ibid. 15, 549 (1982); K. Kremer, A. Baumgärtner, and K. Binder, J. Phys. A 15, 2879 (1982); A. Baumgärtner, J. Phys. 43, 1407 (1982); C. Aragao de Carvalho, S. Caracciolo, and J. Fröhlich, Nucl. Phys. B 215, 209 (1983); S. Havlin and D. Ben-Avraham, J. Phys. A 15, L317 (1982); Phys. Rev. A 26, 1728 (1982); Z. Alexandrowicz, Phys. Rev. Lett. 50, 736 (1983); H. Meirovitch, J. Chem. Phys. 79, 1 (1983).

${ }^{24}$ The method we use was inspired by the lectures given by $M$. H. Kalos in Saclay in 1981.

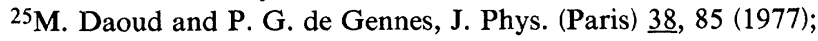
A. J. Guttman and S. G. Wittington, J. Phys. A 11, L107 (1978).

${ }^{26}$ R. Jullien, M. Kolb, and R. Botet, J. Phys. (Paris) 45, 395 (1984).

27P. Meakin, Phys. Rev. B $\underline{28}, 5221$ (1983). 